\title{
Questionnaire Survey about the Reasons of Young People Migrating to the Tsunami-hit Area
}

\author{
Kayo Okabe ${ }^{\mathrm{a}, *}$, Mayumi Dan ${ }^{\mathrm{b}}$, Chikako Nakayama ${ }^{\mathrm{c}}$ \\ ${ }^{a}$ LatLng cooperation, Research, Japan, kayo.okabe@latlng.jp \\ ${ }^{\mathrm{b}}$ Human Science, Tokiwa University, Japan, mdan@tokiwa.ac.jp \\ ${ }^{\mathrm{c}}$ Tokyo University of Foreign Studies, Japan, nakac@tufs.ac.jp
}

Keywords: Tsunami area, young migrants, use of the Internet, face-to-face relationship

Abstract:

This abstract illustrates major results obtained from the questionnaire survey carried out in Kesennuma on October 2018.

We conducted a questionnaire survey focusing on the lifestyles of young migrants living in Kesennuma. The questionnaire was carried out through the Internet using the Google Form. The questionnaire consists of three parts: 1) attitude toward work, income and time management, 2) use of the Internet and 3) interaction with local people.

Kesennuma is the region where greatly damaged by the tsunami caused by the Great East Japan Earthquake occurred in 2011. Many volunteers visited this tsunami-hit area and spent days in reconstructing the area with the local people. Among those volunteers, a certain number of young people decided to migrate from other parts of Japan to the tsunami damaged area. Some left their jobs and found a new job in this area, some after graduating universities started new business, and some returned to their parents' home to help them. Since then, they have been working continuously in many ways, with the local people in order to revitalize the tsunami-hit area, especially in Kesennuma.

The objective of the questionnaire was to find out the reasons why those young people decided to migrate from other parts of Japan and settle down in this local area.

The questionnaire was distributed to those young migrants in Kesennuma area who contacted with the Kesennuma Centre for Migration and Settlement. We received answers from 108 young migrants, and the response rate of the questionnaire was $87 \%$. This number is extremely high compared to common questionnaire response percentage.

We focused on the three topics of the questionnaire, namely Q1: Are you satisfied with your life here?, Q2: How many hours do you use the Internet per day? Q3: How far do you visit your local acquaintances?

Q1: Are you satisfied with your life here?

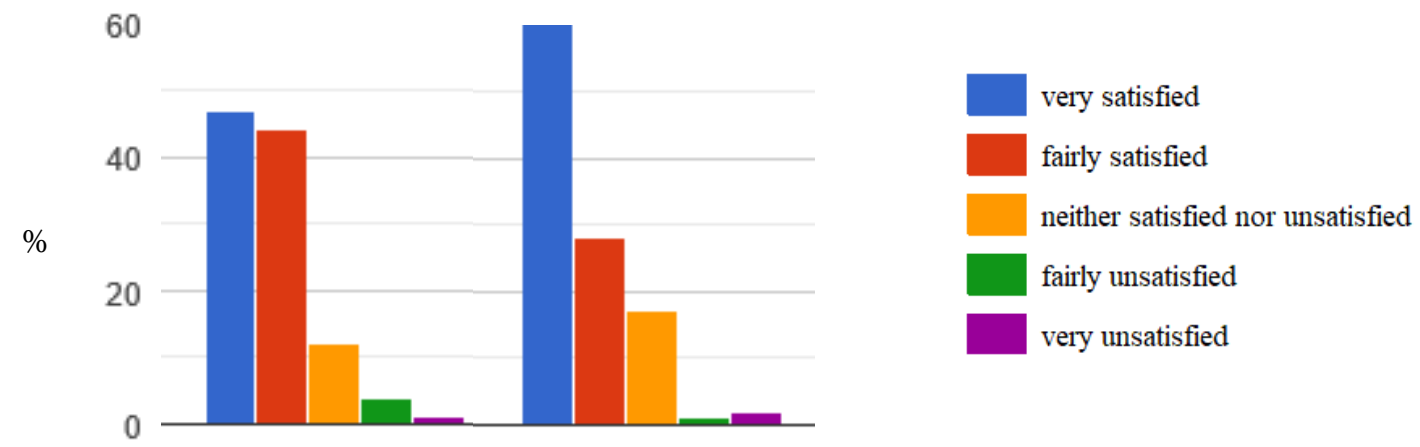

Figure 1 Satisfaction chart (left panel: as a whole, right pane: social relationship with local people)

As for life as a whole here, the percentage of those answered "very satisfied" and "fairly satisfied" reached $89 \%$ of the respondents. In addition, we found that $57 \%$ of respondents answered "very satisfied" with the social relationship with local people.

Q2: How many hours do you use the Internet per day besides your office hours?

The answers to this specific question are shown in Figure 2.
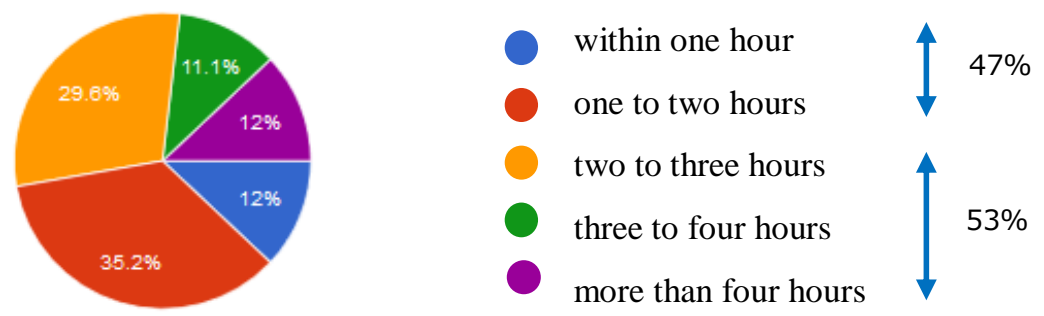
Figure 2 Hours young migrants use the Internet per day besides their office hours

First of all, the very high response rate of the questionnaire clearly tells that these young migrants were well connected with each other through the Internet. Fifty-three percentage of them answered that the average hours of using the Internet per day is more than two hours besides their usage at their work. Fifteen percentage of them have more than 701 followers to their Facebook, this percentage appears to be much higher than an average number.

This heavy use of the Internet is very contrasting to the fact that at the same time, they answered that they enjoyed and valued very highly the face-to- face social relationship with local people.

Q3: How far do you visit your close local acquaintances?

The above two facts motivated us to ask, how often and how far they travelled to visit their nearest local acquaintances. Surprisingly enough, $44 \%$ of them answered that they had daily interaction with local acquaintances such as visiting each other and had chat over a cup of tea, not only exchanging a few words. If we add those who visited local acquaintances two or three times a week, the percentage exceeds 65 . It is easy to imagine that they were very much involved with the local people and the area.

The average walking time to the nearest local acquaintance was two minutes and forty-three seconds, while those going by car, their average travel time was ten minutes and six seconds.

In order to see the spatial interaction between migrants and locals people, we tried to obtain their home addresses. But due to the protection of personal information, we obtained the location data of centres of the districts where they resided and a few of the home addresses. Figure 3 illustrates the nearest local acquaintance distribution with the buffer zone of 217 metres from each location. Note that the travel distance was estimated from [two minutes and forty-three seconds] multiplied by [ 80 metres: an average walking time per minute].

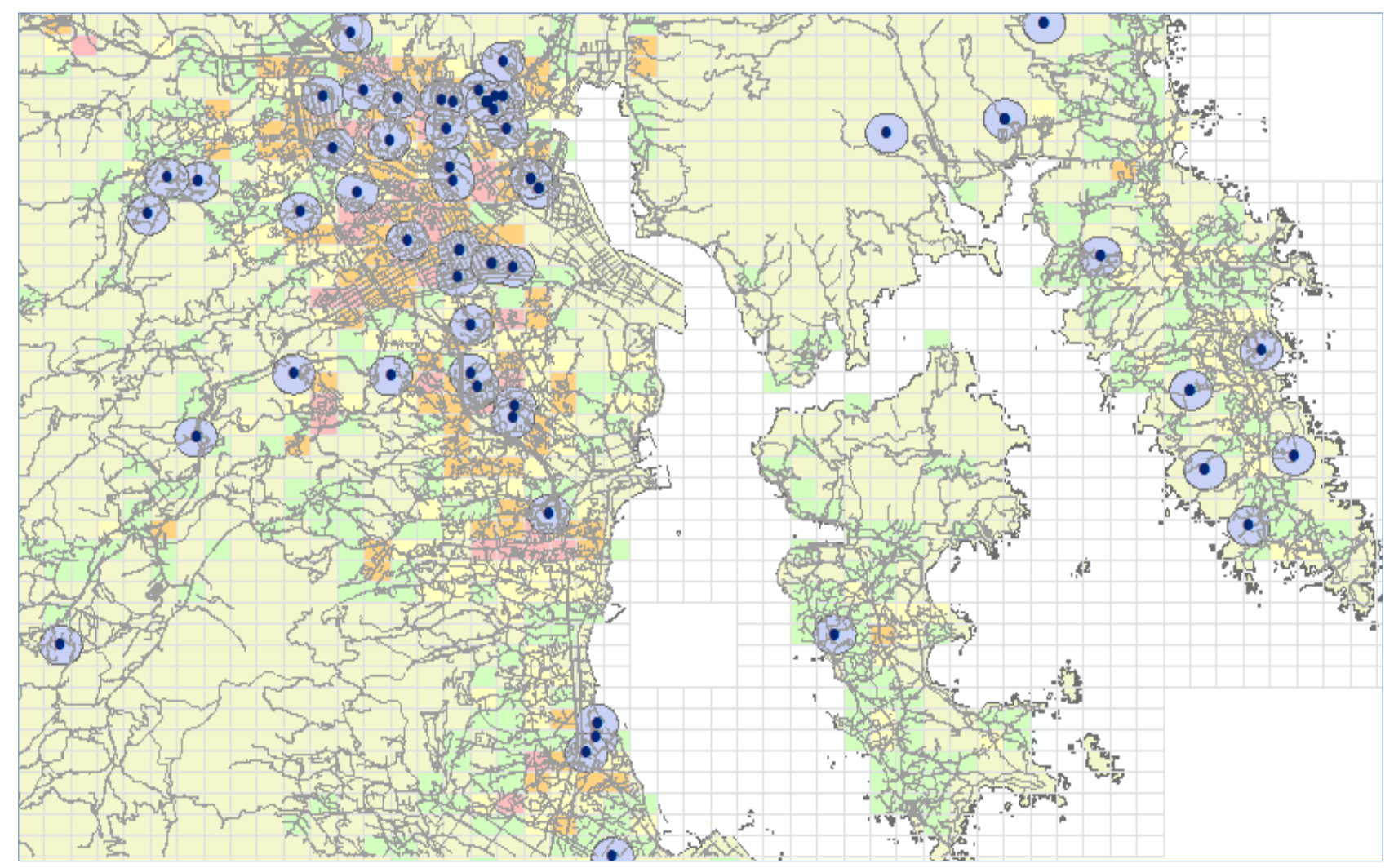

Figure 3 Spatial distribution of the centre of districts where respondents reside with 217 metres buffer zone

From the above results, we conclude that two aspects are important for young migrants' preferable daily life, namely, 1) local and intimate connection with local people, and 2) global connection with many friends through the Internet. These two aspects are contrasting to each other, but both are indispensable at the same time.

As for the GIS map, we use National Geographic Base Map downloadable from the Geospatial Information Authority of Japan, Japanese GSI website for English is http://www.gsi.go.jp/ENGLISH/index.html. Also for the census data, we downloaded from statistics GIS of e-Stat, a portal site for Japanese Government Statistics whose English website is https://www.e-stat.go.jp/en.

Acknowledgement: This questionnaire survey is part of a project supported from the Suntory Foundation on Collaborative Research on Community Cultural Activities. We thank the Foundation. 